$63^{\text {ème }}$ Congrès de la SFCO, 03017 (2015)

DOI:10.1051/sfco/20156303017

(C) Owned by the authors, published by EDP Sciences, 2015

\title{
Agénésie des incisives latérales maxillaires. Prise en charge pré-implantaire et implantaire
}

\author{
Sage $\mathrm{PO} * *, * * *$, Offerle $\mathrm{J}-\mathrm{M}^{*, * *, * * *}$, Keller $\mathrm{P} * * * * * * *$ \\ * Unité de Chirurgie Buccale-Implantologie, Hôpitaux Universitaires de Strasbourg, Strasbourg \\ ** Cabinet de Chirurgie Buccale, 26a avenue de la Forêt noire, Strasbourg \\ *** Clinique de Chirurgie Orale, Ortenau Klinikum, Kanzmattstraße 2a, 77694 Kehl, Allemagne
}

Mots Clés : agénésie dentaire, greffe osseuse autogène, implant dentaire.

Introduction: Les agénésies des incisives latérales maxillaires représentent 22,9\% des agénésies dentaires (Polder et al. 2004). Lors d'une prise en charge implantaire, une coordination entre orthodontiste et dentiste est indispensable. La mise en place d'implants dentaires suppose un volume osseux suffisant. Il est souvent nécessaire de recréer ce volume à l'aide d'une technique de reconstruction osseuse.

But : L'objectif de cette présentation est de faire le point sur la prise en charge chirurgicale, préimplantaire et implantaire d'agénésies d'incisives latérales maxillaires. En fonction du défaut initial, une greffe osseuse autogène, ou une régénération osseuse guidée (ROG), avec une implantation immédiate ou différée est à planifier. Il faut également dans certains cas aménager les tissus mous lors de l'implantation ou pendant le second temps chirurgical.

Matériel et méthode: Douze cas d'agénésie d'incisives latérales maxillaires chez 8 patients (4 cas d’agénésies bilatérales) ont été séléctionnés. Une greffe osseuse autogène a été réalisée sur 6 sites. Nous avons effectué une ROG pour 3 agénésies. Une membrane non résorbable avec de l'os autogène a été utilisée dans un cas. Deux agénésies n’ont pas nécessité de renforcement osseux. Pour 7 agénésies, un lambeau pédiculé palatin a été réalisé lors de la mise en place des implants. Les implants posés sont 10 implants XiVE (DENTSPLY Implants) de 3,0 mm, 1 implant XiVE de 3,4 mm de diamètre et 1 implant Ankylos (DENTSPLY Implants) de 3,5 mm de diamètre.

Résultats : Tous les implants se sont ostéo intégrés. Des couronnes provisoires ont été utilisées sur 8 sites avant la mise en place des couronnes définitives. Une nécrose palatine d'un lambeau pédiculé a été observée chez un patient fumeur, sans conséquence sur l'implant. Le contrôle clinique et radiologique à 1 an a montré une stabilité osseuse et gingivale péri-implantaire chez tous les patients traités.

Discussion : Certains auteurs préconisent une fermeture précoce de l'espace (Rosa et al. 2010) dans le cas d'agénésies d'incisives latérales maxillaires. La solution implantaire reste un procédé de choix pour le confort et l'esthétique du patient. Dans le cas de l'ouverture orthodontique de l'espace une diminution du volume osseux peut survenir (Uribe et al. 2013). La perte osseuse provoquée par l'agénésie dentaire et le faible espace inter-radiculaire ne doivent pas être considérés comme un frein au traitement implantaire. La technique d'aménagement sera fonction du défaut osseux et gingival.

This is an Open Access article distributed under the terms of the Creative Commons Attribution License 4.0, which permits unrestricted use, distribution, and reproduction in any medium, provided the original work is properly cited. 
Conclusion: Une prise en charge multi-disciplinaire est indispensable pour une bonne gestion d'agénésie des incisives latérales maxillaires. Le bon choix de la technique de reconstruction permet la réalisation d'un traitement prothétique de qualité, sans compromis esthétique pour le patient.

Nom et adresse du conférencier

Pierre Olivier SAGE

24 rue des moulins

67000 Strasbourg (France)

sage.pierre.olivier@gmail.com 